\title{
BLAST LOAD ASSESSMENT USING HYDROCODES
}

\author{
Bibiana Luccioni*, Daniel Ambrosini $^{\dagger}$ \\ * Instituto de Estructuras \\ Universidad Nacional de Tucumán, CONICET \\ Av. Roca 1800 (4000) S.M. de Tucumán, Argentina \\ e-mail: bluccioni@herrera.unt.edu.ar \\ ${ }^{\dagger}$ Facultad de Ingeniería \\ Universidad Nacional de Cuyo, CONICET \\ (5500) Mendoza, Argentina \\ e-mail: dambrosini@uncu.edu.ar
}

Key words: Blast loads, pressure, reflection, wave propagation, hydrocodes.

\begin{abstract}
The evaluation of pressures and impulses produced by blast loads with the aid of hydrocodes is studied in this paper. Numerical results are compared with those obtained with existing analytical expressions for different scaled distances and boundary conditions. In particular, the capacity of both methods to capture multiple reflections of the blast load is analyzed. The effects of mesh size on pressure and impulse distribution are also studied. Some interesting conclusions regarding the determination of the best mesh size for calculation of actual events are obtained. The convenience of using mesh refinement close to the explosion zone is also studied.

Finally, the analysis of blast load in the case of the AMIA (Israel-Argentina Mutual Association) building attack occurred in Buenos Aires, Argentina in July 1994 is presented. A computational dynamic analysis was carried out over the congested urban environment that corresponds to the opposite rows of buildings of a block, in the same street. The results obtained for different positions of the explosive charge are presented and compared.
\end{abstract}




\section{INTRODUCTION}

The design and construction of public buildings to provide life safety in the face of explosions is receiving renewed attention from structural engineers ${ }^{1,2,3}$. For many urban settings, the proximity to unregulated traffic brings the terrorist threat to or within the perimeter of the building. For these structures, blast protection has the modest goal of containing damage in the immediate vicinity of the explosion and the prevention of progressive collapse.

The assessment of blast loading effects on structures is required for the design of structures to withstand explosions. In this way, the first stage of the analysis is the accurate and reliable evaluation of the pressures and impulses acting on the structure.

On the other hand, when a blast attack has already occurred, a very important issue is the determination of the location of the focus of the explosion and the mass of the explosive used. A useful tool to achieve this objective is the evidence of the crater generated by the explosion. However, in many cases, the crater remains lost under the parts of the destroyed structures and it is almost impossible to reconstruct its plan. Additionally, the mass of explosive obtained from crater dimensions has a significant spread $^{4,5}$.

Historically the analysis of explosions either has predominantly involved simplified analytical methods ${ }^{4,6,7}$ or has required the use of supercomputers for detailed numerical simulations. With the rapid development of computer hardware over the last decades, it has become possible to make detailed numerical simulations of explosive events in personal computers, significantly increasing the availability of these methods. On the other hand, new developments in integrated computer hydrocodes complete the tools necessary to carry out the numerical analysis successfully.

Important effects such as multiple blast wave reflections, the mach effect, rarefactions, and the negative phase of the blast wave can be readily modeled in computational fluid dynamics (CFD) codes. Simplified analytical and semi-empirical techniques many times ignore such phenomena. Thus, modeling groups of buildings in congested city centers can be treated thoroughly only by the use of sophisticated CFD numerical calculations ${ }^{8}$. In this paper, the program AUTODYN-3D ${ }^{9}$, which is a "hydrocode" that uses finite difference, finite volume, and finite element techniques to solve a wide variety of non-linear problems in solid, fluid and gas dynamics, is used for these purposes.

The distribution of pressures and impulses generated by a blast loading in the congested urban environment is described in this paper. The behavior of blast waves in this type of geometry is both difficult to predict and of great importance in assessing explosion effects on buildings and people.

It is well known that the accuracy of numerical results is strongly dependent on the mesh size used for the analysis. On the other side, the mesh size is also limited by the dimensions of the model and the computer capacity. One of the major features in the numerical simulation of blast wave propagation in large urban environments is the use of an adequate mesh size. The effect of mesh size for different boundary conditions is addressed in this paper. 


\section{GENERATION OF BLAST LOADING}

The analysis of the blast wave propagation was performed in two stages. The first part of the analysis consists of the simulation of the explosion itself, from the detonation instant, and the second part consists of the propagation analysis of the blast wave generated by the explosion.

The use of symmetry conditions allows the spherical portion of the blast wave expansion to be represented by a spherical model. This is achieved by a one-dimensional (1D) mesh using spherical symmetry. The number of cells required to produce accurate solutions is greatly reduced when compared with a full 3D model. When the spherical blast wave begins to interact with obstacles, the flow becomes multi-dimensional. However, before this time the 1D solution can be imposed, or remapped, onto a specific region of the multi-dimensional model. The $3 \mathrm{D}$ calculation can then proceed from that point.

\section{BLAST WAVE PROPAGATION}

The numerical analysis of the blast wave propagation for different boundary conditions and mesh sizes is presented in this section.

\subsection{Free field explosion of spherical charges}

When a condensed high explosive is detonated, a blast wave is formed. It is characterized by an abrupt pressure increase at the shock front, followed by a quasi exponential decay back to ambient pressure and a negative phase in which the pressure is less than ambient pressure. The pressure-time history of a blast wave is often described by exponential functions such as Frielander's equation ${ }^{7}$, which has the form

$$
p(t)=p_{o}+p_{s}\left[1-\left(t-t_{a}\right) / T_{s}\right] \exp \left[-b\left(t-t_{a}\right) / T_{s}\right]
$$

where $\mathrm{t}$ is the time, $p_{o}$ is the ambient pressure, $p_{s}$ is the peak overpressure, $T_{s}$ is duration of the positive phase, $t_{a}$ is the arrival time and $\mathrm{b}$ is a positive constant called the waveform parameter that depends on the peak overpressure.

The most widely used approach to blast wave scaling is Hopkinson's law ${ }^{6}$ which establishes that similar explosive waves are produced at identical scaled distances when two different charges of the same explosive and with the same geometry are detonated in the same atmosphere. Thus, any distance $\mathrm{R}$ from an explosive charge $\mathrm{W}$ can be transformed into a characteristic scaled distance $\mathrm{Z}$,

$$
Z=R / W^{1 / 3}
$$

where $\mathrm{W}$ is the charge mass expressed in kilograms of $\mathrm{TNT}^{10}$. The use of $\mathrm{Z}$ allows a compact and efficient representation of blast wave data for a wide range of situations.

There exist many solutions for the wavefront parameters from both numerical solution and experimental measurements $6,4,7$. The results are usually presented in graphics, tables or equations based on experimental or numerical results as the tables given by Kinney and 
Graham $^{4}$ or the following equations presented by Smith and Hetherington ${ }^{7}$,

$$
\begin{array}{ll}
p_{s}=1407.2 / Z+554.0 / Z^{2}-35.7 / Z^{3}+0.625 / Z^{4}[\mathrm{kPa}] & 0.05 \leq \mathrm{Z} \leq 0.3 \\
p_{s}=619.4 / Z-32.6 / Z^{2}+213.2 / Z^{3}[\mathrm{kPa}] & 0.3 \leq \mathrm{Z} \leq 1.0 \\
p_{s}=66.2 / Z+405.0 / Z^{2}-328.8 / Z^{3}[\mathrm{kPa}] & 1.0 \leq \mathrm{Z} \leq 10
\end{array}
$$

The accuracy of predictions and measurements in the near field is lower than in the far field, probably due to the complexity of blast phenomenon ${ }^{7}$.

In order to study the free propagation of blast waves in air, a $5 \mathrm{~m}$ by $5 \mathrm{~m}$ by $10 \mathrm{~m}$ volume of air was numerically modeled with four different mesh sizes: $50 \mathrm{~mm}, 100 \mathrm{~mm}, 250 \mathrm{~mm}$ and $500 \mathrm{~mm}$, see Figure 1. A three dimensional Euler $\mathrm{FCT}^{9}$ (higher order Euler processor) subgrid was used for the air. The Euler-FCT processor has been optimized for gas dynamic problems and blast problems and it is much more efficient in comparison with a general purpose high resolution Euler processor. FCT stands for Flux Corrected Transport ${ }^{11}$. With FCT a high order solution is computed wherever this is possible in the flow field. The high order solution fluxes are corrected in the regions of shocks using a low order reference (transported and diffused) solution.

In order to simulate a free field explosion, flow out of air was allowed in all the borders of the models.

The results of the 1D analysis of explosive spherical charges ranging from $1 \mathrm{~kg}$ to $500 \mathrm{~kg}$ were mapped in the 3D air models at the position $P(x=y=z=2.5 \mathrm{~m})$, indicated with a star in Figure 1.

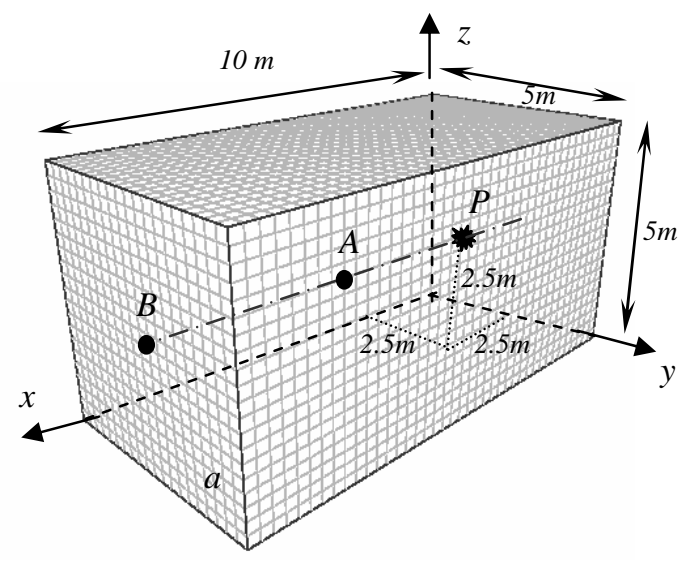

Figure 1. Model for the study of blast wave free propagation

Figure 2 shows a pressure-time history curve obtained for point $A$ $(x=5.5 \mathrm{~m}, y=z=2.5 \mathrm{~m})$, located at $3 \mathrm{~m}$ from a $100 \mathrm{~kg}$ of TNT explosive charge. It can be 
seen that the curves resemble that one described by Equation (1), but there are important differences among the peaks overpressures obtained for the different mesh sizes. It me be noted that, as the mesh is refined, the difference between the results for the different mesh sizes are reduced showing the convergence of numerical results.

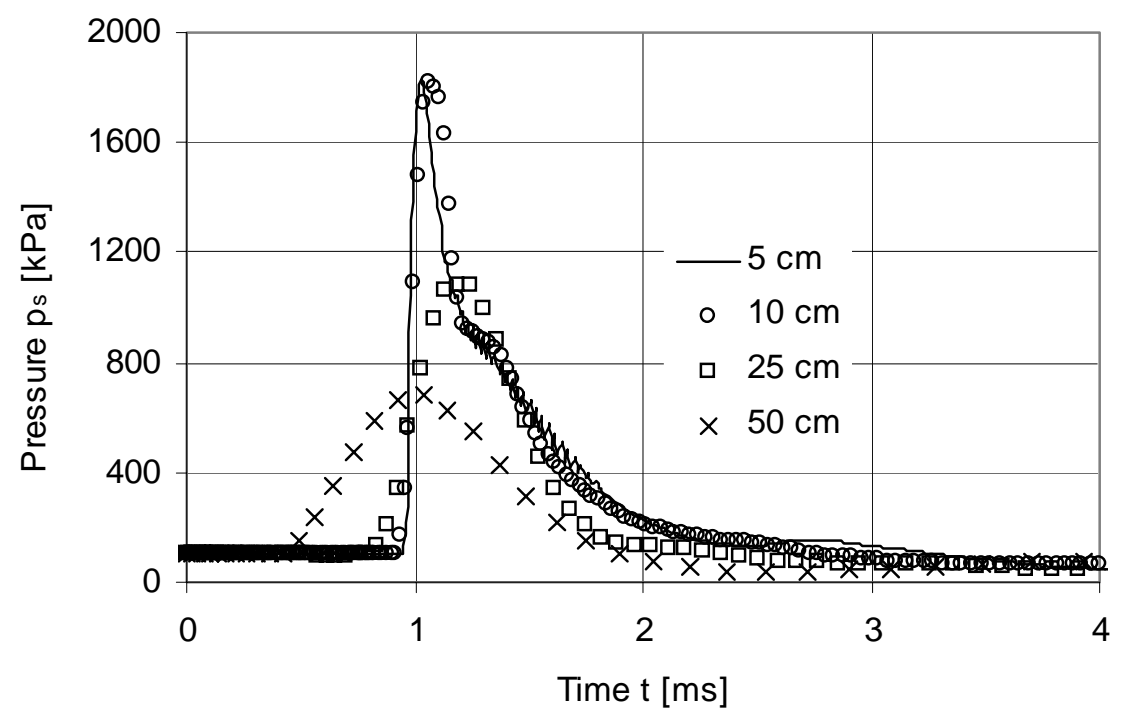

Figure 2. Pressure-time history.

Numerical results for the peak overpressure related to the ambient for different scaled distances are compared with those obtained with empirical equations and tables ${ }^{4,6,7}$ in Figure 3. For high scaled distances the results obtained with the finest mesh are much closer to empirical ones. As the scaled distance decreases, numerical results depart from empirical ones. Nevertheless, it may be noted that the accuracy of empirical relations in the near field is not guaranteed ${ }^{7}$. The difference with empirical values is more marked for coarser meshes that give lower values for the peak overpressure, but results tend to converge as the mesh is refined. The results corresponding to the meshes of $5 \mathrm{~cm}$ and $10 \mathrm{~cm}$ are almost coincident. It can be concluded that the mesh of $100 \mathrm{~mm}$ gives an accurate solution to the problem.

The comparison of maximum impulses is presented in Figure 4. For high scaled distances the impulses obtained with the finest mesh are much closer to empirical ones. As the scaled distance decreases numerical results depart from empirical ones and the tendency is not as defined as in the case of pressures. 


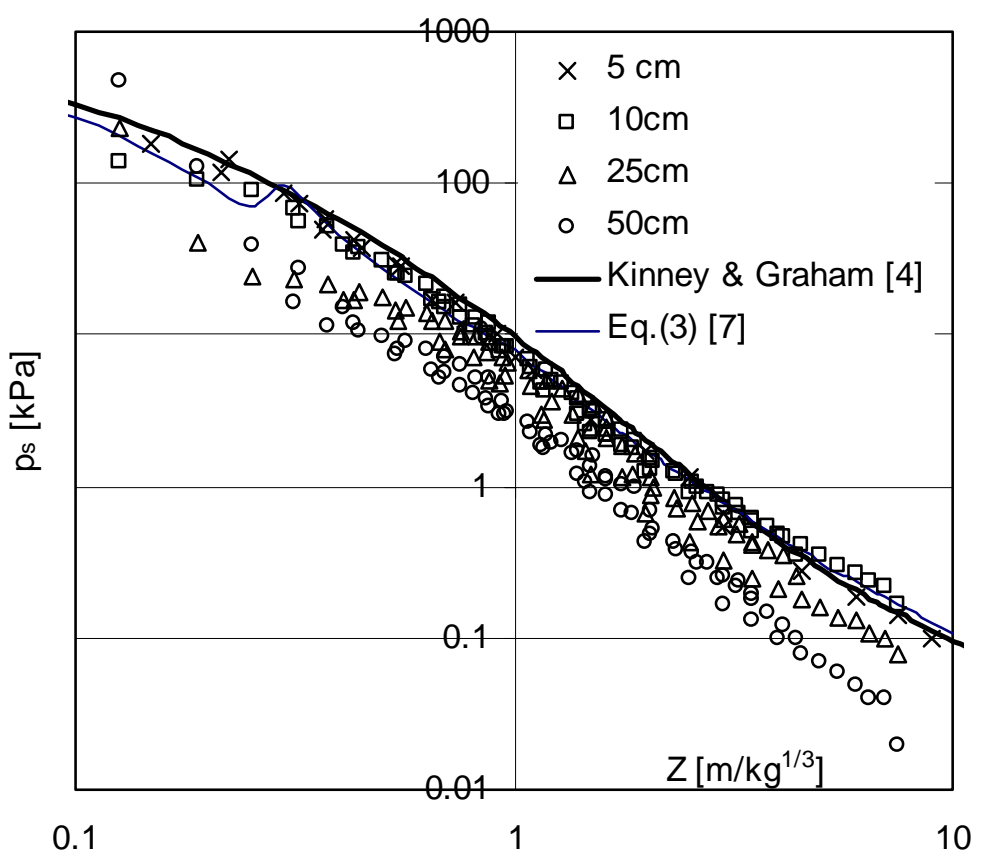

Figure 3. Peak side-on overpressures as a function of scaled distance

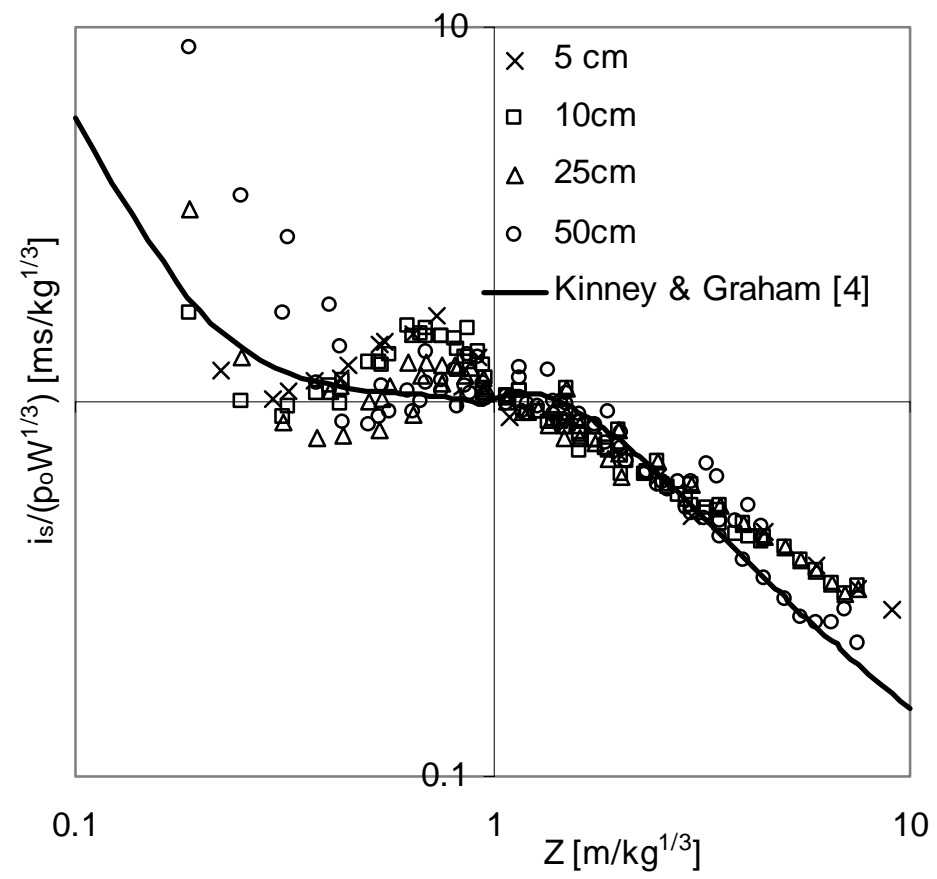

Figure 4. Peak side-on impulses as a function of scaled distance 


\subsection{Reflected waves with normal incidence}

When the blast waves encounter an infinite large wall on which they impinge at zero angle of incidence, they are normally reflected. All flow behind the wave is stopped and pressures are considerably greater than side on.

The peak reflected pressure $p_{r}$ can be obtained from Rankine Hugoniot relationships for an ideal gas and results ${ }^{7}$,

$$
p_{r}=2 p_{s}\left(7 p_{o}+4 p_{s}\right) /\left(7 p_{o}+p_{s}\right)
$$

Lacking of more accurate prediction methods the reflected impulse can be estimated by assuming similarity between the time histories of side-on overpressures and normally reflected overpressure. This assumption gives ${ }^{6}$,

$$
i_{r} / i_{s} \approx p_{r} / p_{s}
$$

where $i_{s}$ is the peak side-on impulse and $i_{r}$ is the peak reflected impulse.

In order to study the normal reflection of blast waves the same numerical models presented in Figure 1 were used, but this time an infinite rigid surface was defined on face $a$, normal to the wave propagation direction. The comparison of peak reflected overpressures numerically obtained for point $B(x=10 m, y=z=2.5 m)$, for different scaled distances and different mesh sizes, and those obtained with Equation (4) is presented in Figure 5. Reflected impulses are compared in Figure 6. The same conclusions than in the case of free propagation values can be stated but, in this case, the difference in impulse values for the different mesh sizes is lower.

\subsection{Regular and Mach reflection}

The most usual case of loading of large flat surfaces is represented by waves that strike at oblique incidence. For angles of incidence between $0^{\circ}$ and $90^{\circ}$, either regular or Mach reflection occurs depending on incident angle and shock strength ${ }^{6,7}$. The evaluation of reflected pressures resulting form multiple reflections on surfaces with different incidence angle is much complicated and difficult to perform with empirical equations. In this case, the use of numerical methods is more appropriate.

In order to analyze the reflected pressures and impulses produced by this type of problems, the model presented in Figure 7 was used. Faces $a, b$ and $c$ were considered to be infinitely rigid, while air flow out was allowed in the other faces. The dimensions of this model were chosen in order to get conclusions applicable to the example later presented in the paper.

Three different mesh sizes were used: $100 \mathrm{~mm}, 250 \mathrm{~mm}$ and $500 \mathrm{~mm}$. The finest mesh $(50$ $\mathrm{mm}$ ) was not used because it requires too much cells and it has been proved that it gives results almost coincident with those of the $100 \mathrm{~mm}$ mesh. For all the calculations a spherical explosive charge of $300 \mathrm{~kg}$ of TNT with the location indicated as I in Figure 7 was used. 


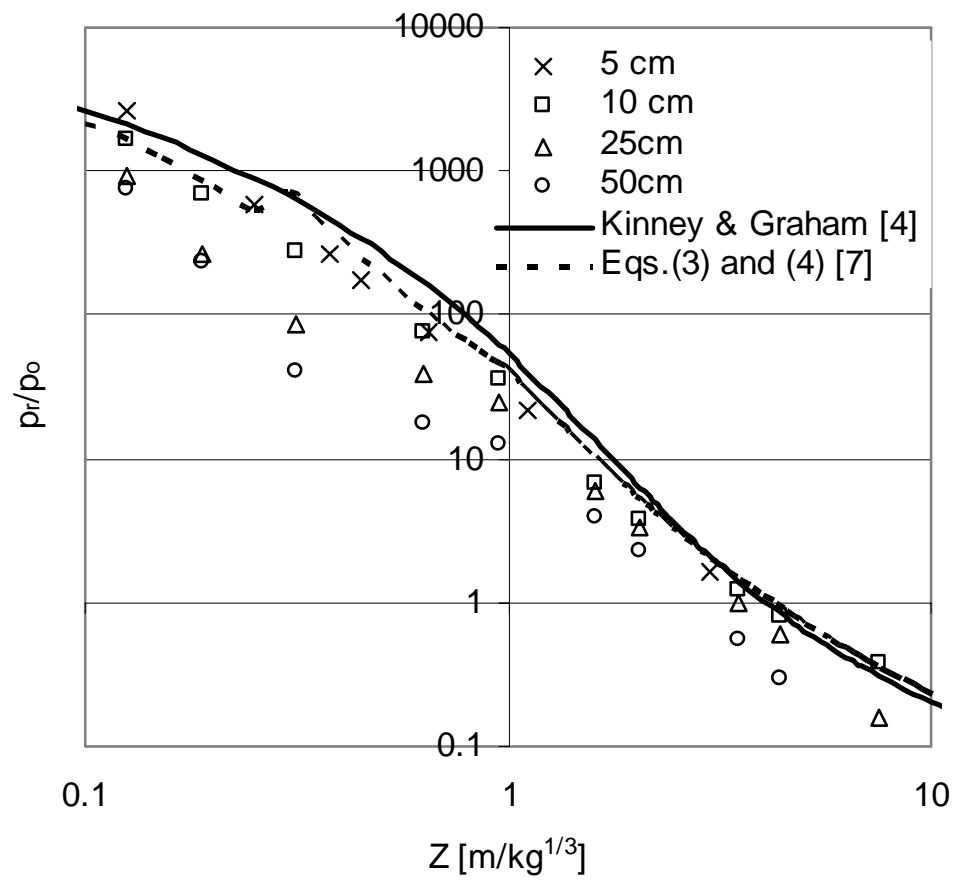

Figure 5. Peak reflected overpressures as a function of scaled distance

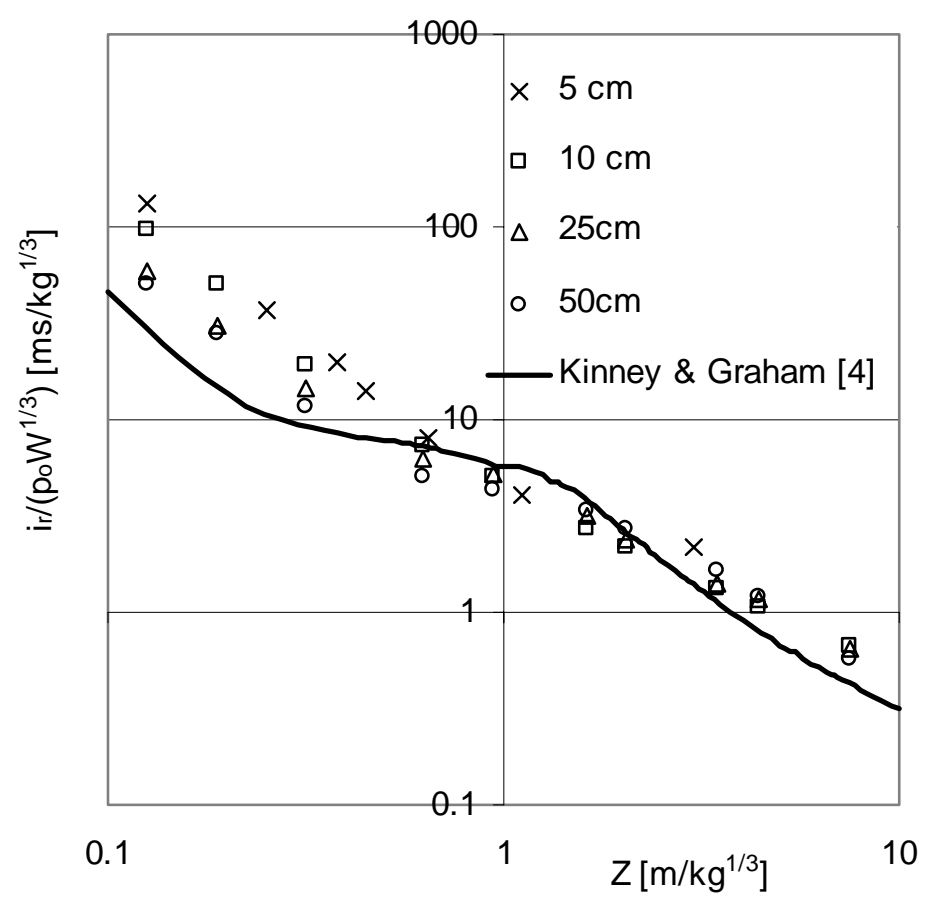

Figure 6. Peak reflected impulses as a function of scaled distance 


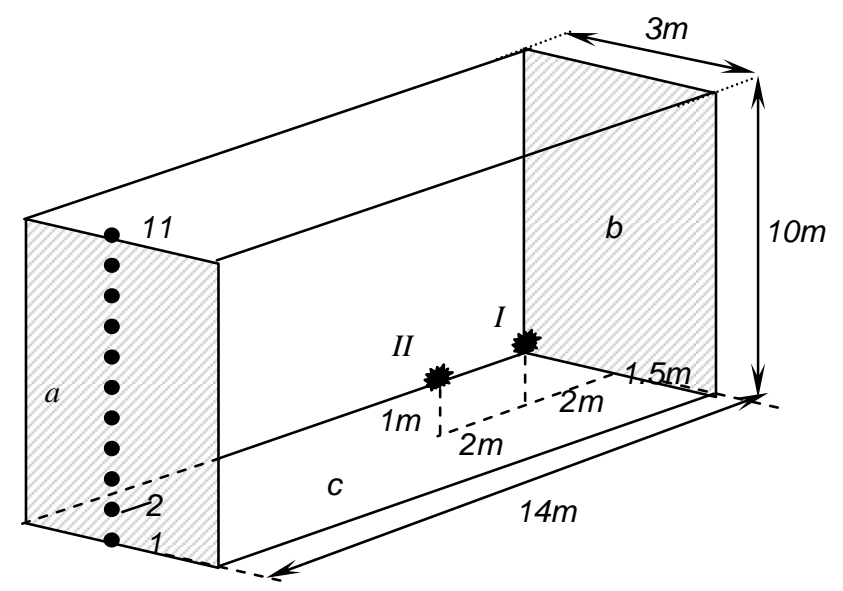

Figure 7. Model for the study of blast wave propagation with oblique reflections

Figure 8 shows the pressure-time history obtained for point 2 (face $a$ in fig. 7) with this model and that obtained for the same model but allowing air flow out in faces $b$ and $c$. Both curves were obtained with the $100 \mathrm{~mm}$ mesh. The sub estimation of the peak reflected pressure when reflection on faces $b$ and $c$ is neglected is clear in Figure 8. The wave resulting from the reflection on face $b$ is also clear in numerical results.

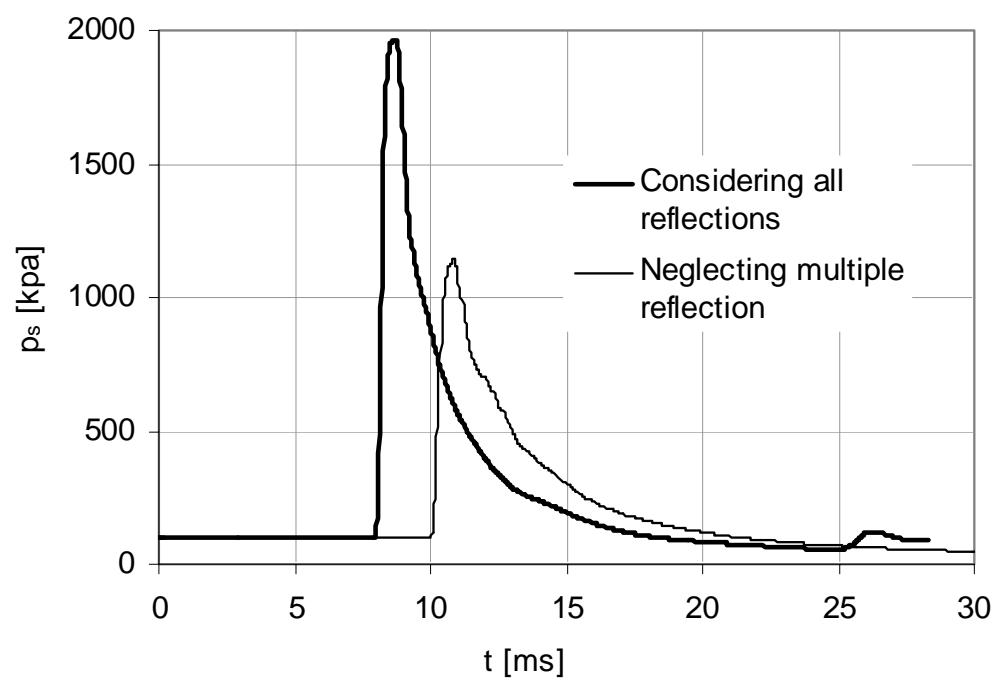

Figure 8. Comparison of pressure-time histories for different reflecting conditions.

Figures 9 and 10 show the values of peak reflected pressure and impulse respectively along a vertical line in face a (see Figure 7) and the comparison with empirical values obtained only considering normal reflection on face $a$. It is clear that both pressure an impulse values are under estimated when the mesh gets coarser. But in even for the $50 \mathrm{~cm} \mathrm{mesh}$, the values are 
greater than those obtained empirically neglecting reflections on surfaces $b$ and $c$.

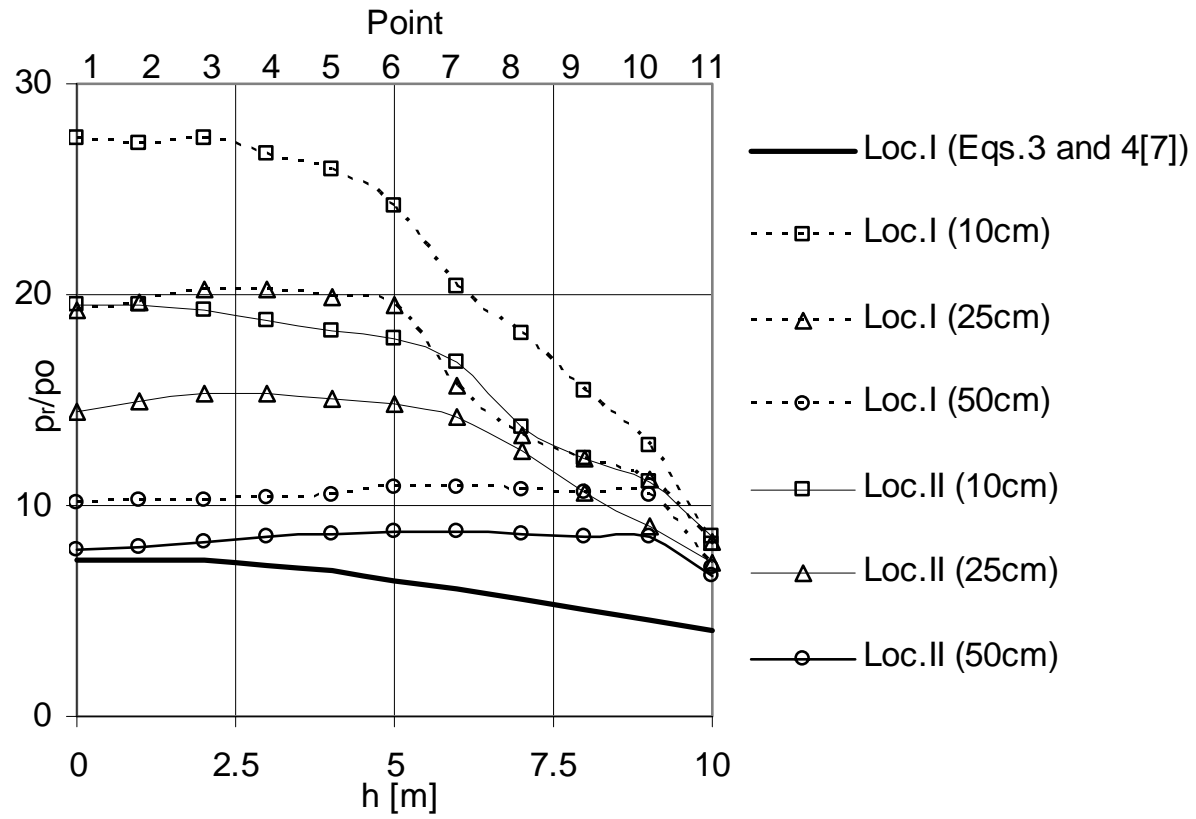

Figure 9. Peak reflected overpressures along a vertical line

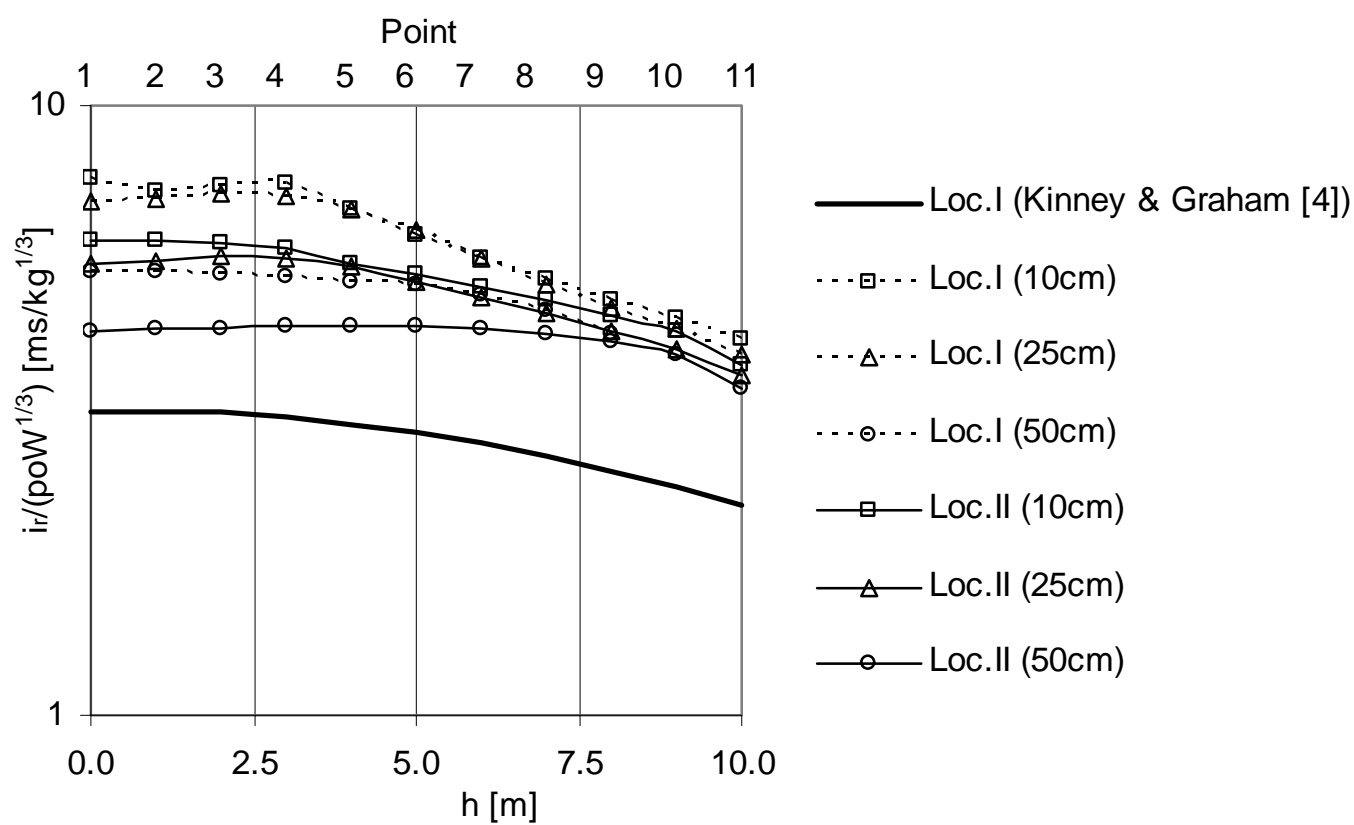

Figure 10. Peak reflected impulses along a vertical line 
Figures 9 and 10 also show the comparison of pressure and impulse distributions along a vertical line when the explosive charge is moved to position II indicated in Figure 8. It is clear that, although coarse meshes underestimate pressures and impulses, the effect of moving the explosive charge is qualitatively captured even by the coarsest mesh.

\section{NUMERICAL SIMULATION OF AN ACTUAL BLAST EVENT IN AN URBAN ENVIRONMENT}

The evaluation of blast loads produced by the explosion that destroyed part of the AMIA (Israel-Argentina Mutual Association) building in the city of Buenos Aires in 1994 is presented in this section. The blast scenario is illustrated in Figure 11 and corresponds to the two rows of buildings of a block, in the opposite sides of the same street.

Many alternatives of mass of explosive and locations were analyzed. 200, 300, 400 and 500 $\mathrm{kg}$ of TNT were used because they are in the medium range of terrorist attacks to buildings. The range of explosive masses used in terrorist attacks is discussed in some papers ${ }^{1,2}$ and it is strongly dependent on how the explosive is supposed to have been transported. The trial locations were inferred from a visual inspection of photographs of damage and result in correspondence with the front of the target building. For sake of clarity, only the results correspondent to $300 \mathrm{~kg}$ of TNT in locations indicated as 1,2 and 3 in the entrance of building 1 in Figure 12 will be shown in this paper.

Due to the large dimensions of the problem analyzed and taking into account that the aim of this study was the comparison of the effects of different locations of the explosive on resulting blast loads on structures, a coarse mesh of $50 \mathrm{~cm}$ was used. Moreover, the model had to be split in two parts (Figure 12) in order to make possible the computational analysis with a PC. Model 1 (380,000 cells) corresponds to the row of buildings where the target building was located and it extends up to the street axis. Model 2 (615,000 cells) corresponds to the row of buildings in the opposite side of the street. The numerical analysis presented in previous section showed that, for the row of buildings closer to the explosive charge, the lost wave reflections on the facades of the buildings of the opposite side of the street are considerable out of phase with respect to the main shock. This fact is due to the relatively long distances involved. However, in the opposite side of the street (Model 2), the coupling between the waves generated by the main shock and that due to the reflection on the target building is important. For this reason, the building facades that constitute the principal source of reflection were taken into account in the model. Particularly, the entrance hall of AMIA building had reflecting surfaces in its laterals but not at the back were there was an iron grating.

The buildings were defined as "unused" regions (AUTODYN 2003) and were considered to behave as rigid surfaces. Air flow out was allowed in the remaining boundaries of the model. 

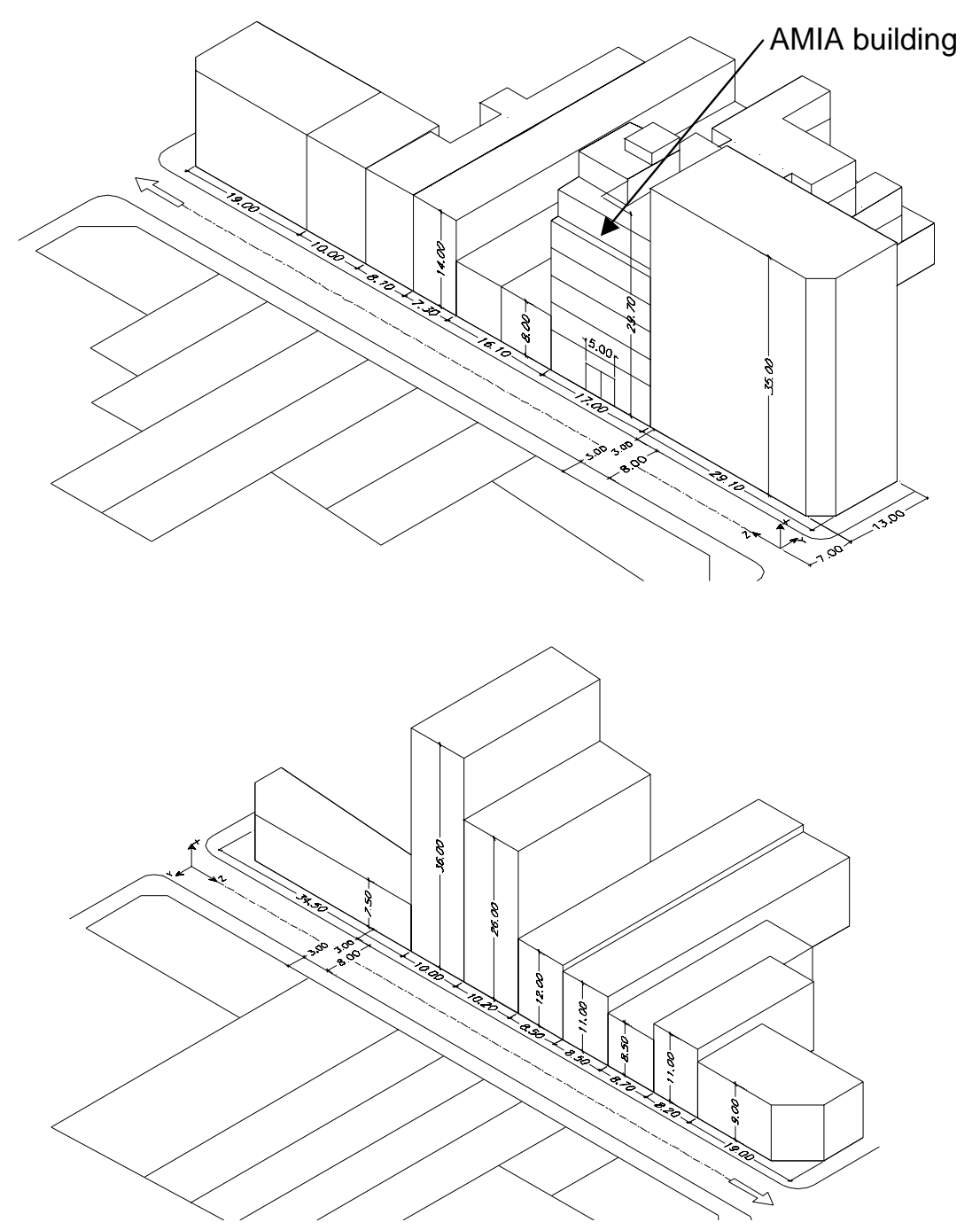

Figure 11. Blast scenario

The curves representing the time history of the reflected pressures and impulses for $300 \mathrm{~kg}$ TNT for location 1 are presented in Figure 13. These records correspond to the target points situated on a vertical line in the center of building 4 (see Figure 12b). The results on the façade of this building were chosen for this analysis because they are more sensitive to the difference of blast load location than the other buildings.

Additionally, the distribution of peak reflected overpressures and impulses with height for building 4, for the different locations are presented in Figure 14. When the explosive charge is moved away from the building analyzed the value of peak overpressure and impulse decreases until certain limit when the pressure begins to increase due to the effects of reflections of the blast wave on the façades of the buildings on the opposite side of the street. These results could have never been achieved through empirical expressions. 
a)
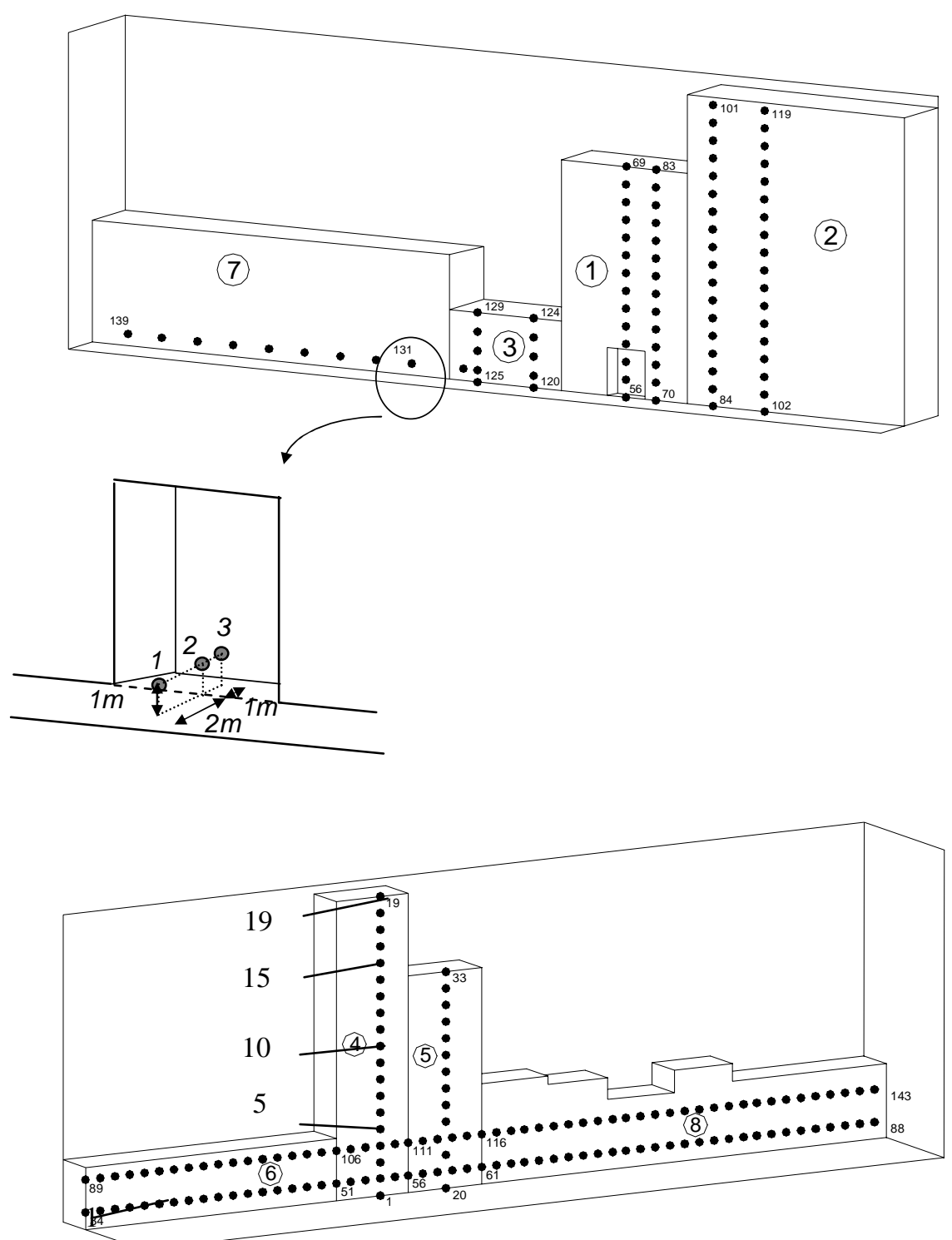

b)

Figure 12. Model for the simulation of an actual blast event a) Part 1, b) Part 2 


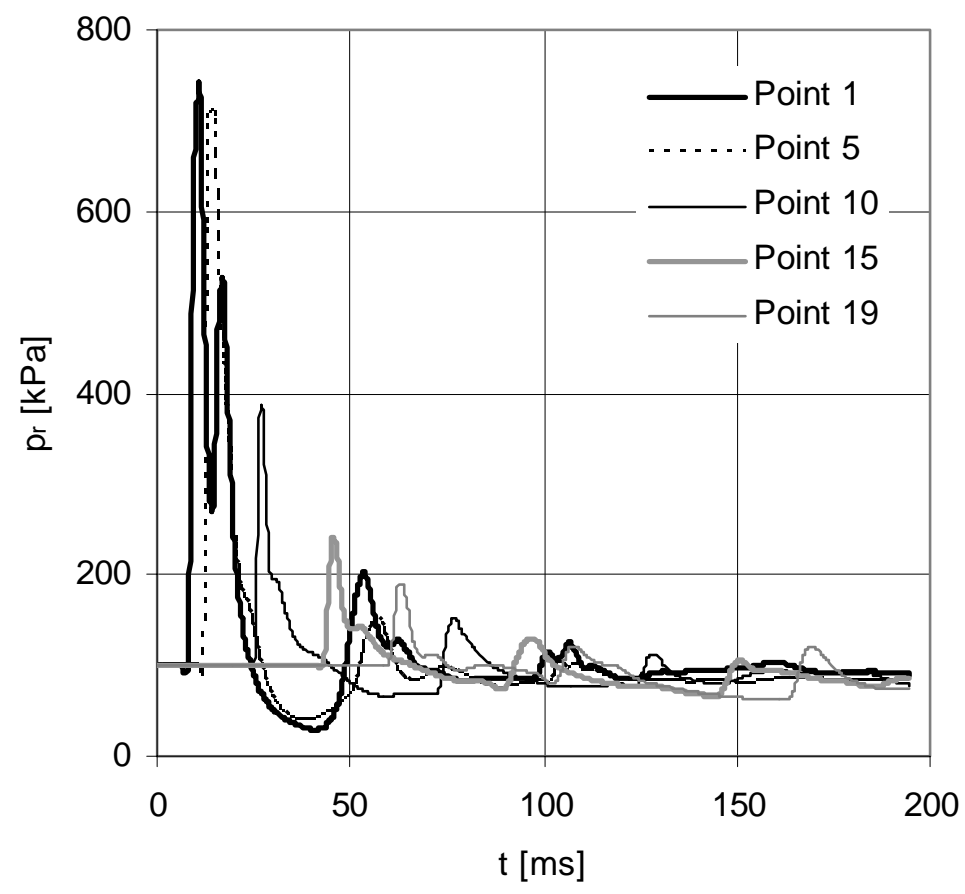

Figure 13. Pressure-time histories for points 1, 5, 10, 15 and 19 (location 1)

\section{CONCLUSIONS}

According to the results presented in the paper, it is clear that, the use of empirical expressions is not enough for the accurate evaluation of incident pressure distributions and associated impulses in complex urban environments. Neglecting reflections and the "mach effect" of the blast wave could lead to important underestimation of the peak values in the far field. Moreover, empirical expressions are not applicable with confidence in the near field because of the complexity of the flow processes involved in forming the blast wave.

For these cases the detailed time-history of side-on and reflected pressures and impulses can accurately be obtained with numerical methods such as hydrocodes. This type of analysis can reproduce not only free blast wave propagation but also normal and oblique reflection occurring on buildings facades.

The accuracy of numerical results is strongly dependent on the mesh size used for the analysis. A $10 \mathrm{~cm}$ mesh is accurate enough for the analysis of wave propagation in urban environments. Nevertheless it may be too expensive to model a complete block with this mesh size. Alternatively, a coarser mesh can be used in order to obtain qualitative results for the comparison of the loads produced by different hypothetical blast events. Even coarse meshes, up to $50 \mathrm{~cm}$ of side, give a good estimation of the effects of moving the location of the explosive charges. 
The difference between numerical results for different mesh size increases with decreasing scaled distances. It may be marked that, even for the coarser mesh of $50 \mathrm{~cm}$ side, the results are more conservative than those obtained with empirical expressions neglecting multiple reflections that take part in actual situations.

Another important feature is that the differences obtained are lesser for the case of impulses than for the case of pressure. This conclusion is important taking into account that, for the range of scaled distances involved in actual urban environments, damage is practically defined by the value of peak reflected impulse.
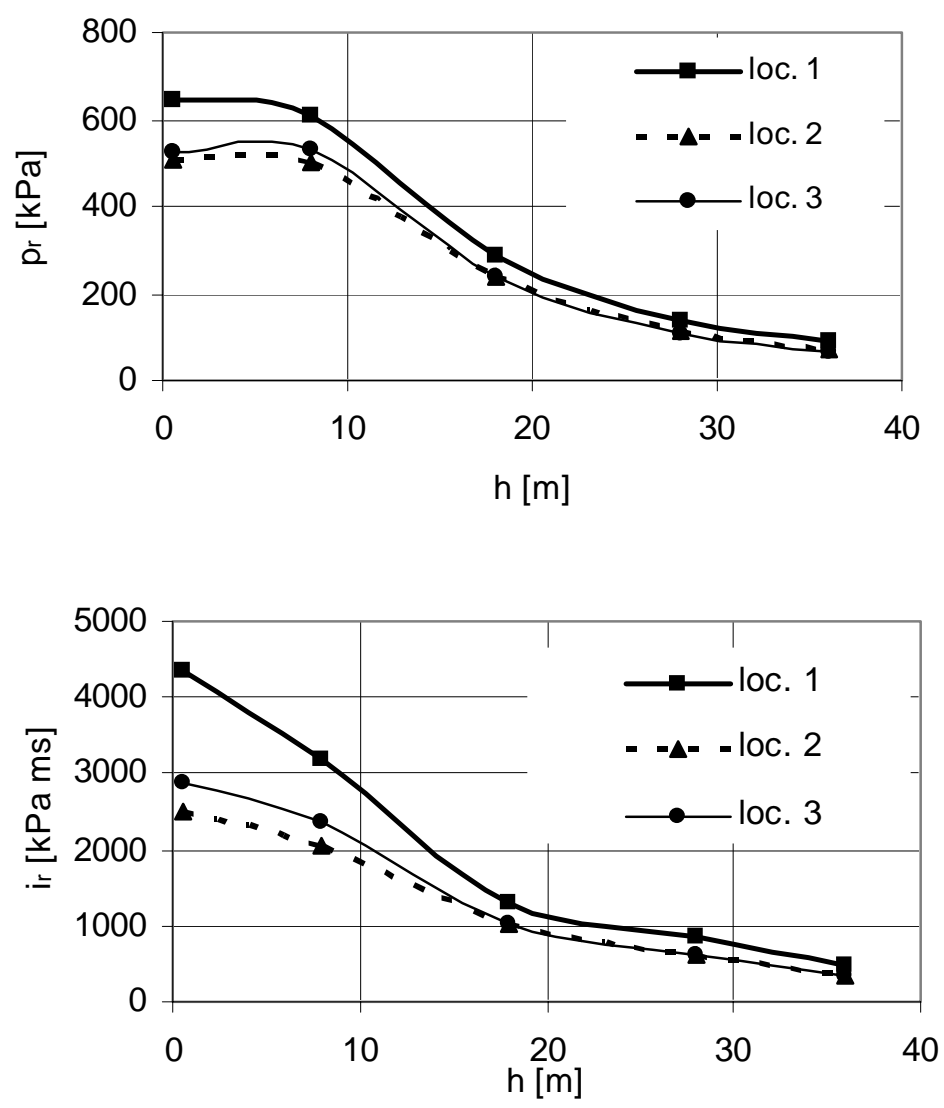

Figure 14. Peak reflected values for different locations of the explosive charge. a) Overpressures, b) Impulses

\section{ACKNOWLEDGEMENTS}

The financial support of the CONICET, the National University of Tucumán and the Argentine Judiciary Board is gratefully acknowledged. Special acknowledgements are extended to Drs. Bence Gerber and Chris X. Quan for the technical support of AUTODYN 
and to Ms. Amelia Campos for the English revision.

\section{REFERENCES}

[1] C.L. Elliot, G. C. Mays and P. D. Smith. "The protection of buildings against terrorism and disorder", Proceedings of Institution of Civil Engineers: Structures \& Buildings, 94, 287-297 (1992).

[2] G. Millington, "Discussion of 'The protection of buildings against terrorism and disorder' by Elliot C.L., Mays G.C. and Smith P.D." Proceedings of Institution of Civil Engineers: Structures \& Buildings, 104, 343-350 (1994).

[3] Committee on Feasibility of Applying Blast-Mitigating Technologies and Design Methodologies from Military Facilities to Civilian Buildings, Protecting Buildings From Bomb DamageNational Academy Press, , Washington (1995).

[4] G. F. Kinney and K.J. Graham, Explosive shocks in air, 2nd Edition, Springer Verlag, Berlin, (1985).

[5] R.D. Ambrosini, B.M. Luccioni, R.F. Danesi, J.D. Riera and M.M. Rocha, "Size of Craters Produced by Explosive Charges on or Above the Ground Surface", Shock Waves, 12(1), 69-78 (2002).

[6] W.E. Baker, P.A. Cox, P.S. Westine, J.J. Kulesz and R.A. Strehlow, Explosion hazards and evaluation, Elsevier, Amsterdam, (1983).

[7] P.D. Smith and J.G. Hetherington, Blast and Ballistic Loading of Structures, Butterworth-Heinemann Ltd, Great Britain, (1994).

[8] P.D. Smith and T.A. Rose, "Blast loading and building robustness", Progress in Structural Engineering and Materials, 4(2), 213-223 (2002)

[9] AUTODYN user's manual-revision 4.3.Century Dynamics Inc., (2003).

[10]S.A. Formby, R.K. Wharton, "Blast Characteristics and TNT Equivalence Values for Some Commercial Explosives Detonated at Ground Level", Journal of Hazardous Materials, 50, 183-198 (1996).

[11]E.S. Oran and J.P. Boris, Numerical Simulation of Reactive Flow, Elsevier, (1987). 